\title{
Pediatric Moyamoya Presenting as a Subarachnoid Hemorrhage from a Ruptured Anterior Cerebral Artery Aneurysm.
}

\author{
Mohammad Hassan A. Noureldine \\ Johns Hopkins University School of Medicine \\ Ibrahim Saikali \\ Lebanese American University \\ Anis Nassif \\ Lebanese American University \\ Rita Chahinian \\ Lebanese American University
}

Ablnawh \$nis arid additional works at: https://jdc.jefferson.edu/neurosurgeryfp Thomas Jefferson University

Part of the Neurology Commons, and the Surgery Commons

\section{$\frac{\text { Let us know how access to this document benefits you }}{\text { See next page for additional authors }}$}

\section{Recommended Citation}

Noureldine, Mohammad Hassan A.; Saikali, Ibrahim; Nassif, Anis; Chahinian, Rita; Sweid, Ahmad; Kikano, Raghid; and Mawad, Michel, "Pediatric Moyamoya Presenting as a Subarachnoid Hemorrhage from a Ruptured Anterior Cerebral Artery Aneurysm." (2019). Department of Neurosurgery Faculty Papers. Paper 114.

https://jdc.jefferson.edu/neurosurgeryfp/114

This Article is brought to you for free and open access by the Jefferson Digital Commons. The Jefferson Digital Commons is a service of Thomas Jefferson University's Center for Teaching and Learning (CTL). The Commons is a showcase for Jefferson books and journals, peer-reviewed scholarly publications, unique historical collections from the University archives, and teaching tools. The Jefferson Digital Commons allows researchers and interested readers anywhere in the world to learn about and keep up to date with Jefferson scholarship. This article has been accepted for inclusion in Department of Neurosurgery Faculty Papers by an authorized administrator of the Jefferson Digital Commons. For more information, please contact: JeffersonDigitalCommons@jefferson.edu. 


\section{Authors}

Mohammad Hassan A. Noureldine, Ibrahim Saikali, Anis Nassif, Rita Chahinian, Ahmad Sweid, Raghid Kikano, and Michel Mawad 
Title: Pediatric Moyamoya presenting as a subarachnoid hemorrhage from a ruptured anterior cerebral artery aneurysm

Running Title: Pediatric Moyamoya-associated cerebral aneurysm

\section{Authors and Affiliations:}

Mohammad Hassan A. Noureldine, M.D., M.Sc. ${ }^{1}$, Ibrahim Saikali, M.D. ${ }^{2, *}$, Anis Nassif, M.D. ${ }^{3}$, Rita Chahinian, M.D. ${ }^{3}$, Ahmad Sweid, M.D. ${ }^{4}$, Raghid Kikano, M.D. ${ }^{3}$, Michel Mawad, M.D. ${ }^{3}$

${ }^{1}$ Department of Neurosurgery, Johns Hopkins University School of Medicine, Institute for Brain Protection Sciences, Johns Hopkins All Children's Hospital, Saint Petersburg, Florida, USA

${ }^{2}$ Division of Neurosurgery, Gilbert and Rose-Marie Chagoury School of Medicine, Lebanese American University, Beirut, Lebanon

${ }^{3}$ Department of Radiology, Gilbert and Rose-Marie Chagoury School of Medicine, Lebanese American University, Beirut, Lebanon

${ }^{4}$ Department of Neurosurgery, Thomas Jefferson University Hospital, Philadelphia, Pennsylvania, USA

\section{*Corresponding author:}

Ibrahim Saikali, MD, FRCSC, Division of Neurosurgery, Gilbert and Rose-Marie Chagoury School of Medicine, Lebanese American University, Beirut, Lebanon

Email: ibrahim.saikali@lau.edu.lb

Mobile: +9613434350

Fax: +9611200816 


\begin{abstract}
Background: The incidence of Moyamoya disease (MMD)-associated intracranial aneurysms ranges from $3 \%$ to $14 \%$ in adult patients, whereas this complication has been rarely reported in children.

Case Description: We herein report the first case of an extremely rare subarachnoid hemorrhage (SAH) presentation of a child with a ruptured anterior cerebral artery (ACA) dissecting aneurysm secondary to a newly discovered, unilateral Moyamoya-like pathology.

Conclusion: MMD-associated aneurysms are extremely rare in children, and hemorrhage may be the initial presentation of the disease. Prompt intervention is essential to exclude the ruptured aneurysm that is at risk of rebleeding due to the persistent hemodynamic stress.
\end{abstract}

\title{
Keywords
}

Pediatric Moyamoya; Aneurysm; Anterior cerebral artery; Subarachnoid hemorrhage

\section{Abbreviations and Acronyms}

ACA: anterior cerebral artery

CT: computed tomography

CTA: CT angiography

DSA: digital subtraction angiography

EDAMS: encephaloduroarteriomyosynangiosis

EDAS: encephaloduroarteriosynangiosis

FLAIR: fluid-attenuated inversion recovery

ICA: internal carotid artery

ICU: intensive care unit

MCA: middle cerebral artery 
MMD: Moyamoya disease

MRI: magnetic resonance imaging

SAH: subarachnoid hemorrhage

STA-MCA: superficial temporal artery-middle cerebral artery 


\section{Introduction}

Moyamoya disease (MMD) is a cerebrovascular pathology characterized by chronic, progressive stenosis of the intracranial internal carotid arteries (ICAs) as well as the proximal portions of their major branches, the anterior cerebral arteries (ACAs) and middle cerebral arteries (MCAs), in association with dilated basal collateral vessels. ${ }^{1}$ The vast majority of patients with MMD present with an ischemic event, although seizures, headaches, chorea and intracranial hemorrhage have been reported as well. ${ }^{2}$ Hemorrhage may be intraparenchymal, mainly in the basal ganglia region and secondary to the rupture of the newly-formed, fragile collateral vessels that are unable to accommodate for the hemodynamic changes associated with the progressive stenosis of larger vessels, intraventricular, and, less likely, subarachnoid. ${ }^{3}$ The major sources of intraventricular and subarachnoid hemorrhage $(\mathrm{SAH})$ are intracranial aneurysms, a two-type classification for which has been proposed: major artery aneurysms and blood flow-related aneurysms ${ }^{4}$. The location of the aneurysm is strongly associated with the hemorrhage type, where major artery aneurysms are more likely to cause SAH and the peripheral, blood flow-related aneurysms more frequently lead to intraparenchymal and intraventricular hemorrhage. ${ }^{4}$ The incidence of MMD-associated intracranial aneurysms ranges from $3 \%$ to $14 \%$ in adult patients, ${ }^{5}$ whereas this complication has been rarely reported in children. Most of the reported hemorrhages in children cases are intraventricular; to our knowledge, only one child presenting with SAH from a posterior communicating artery aneurysm has been reported so far. ${ }^{6}$ We herein report the first case of an extremely rare SAH presentation of a child with a ruptured ACA dissecting aneurysm secondary to a newly discovered, unilateral Moyamoya-like pathology. 


\section{Case Description}

Our patient is a 14-year-old girl with no previous medical, family or trauma history who presented to the emergency department complaining of recent onset of the 'worst headache of her life'. Her neurological examination was pertinent for confusion, vertical nystagmus, and bilateral lateral gaze palsy. Urgent brain computed tomography (CT) scan revealed SAH in the basal cisterns with predilection to the right side (Figure 1-A). Subsequent brain CT angiography (CTA) confirmed the findings, revealing a $4 \mathrm{~mm}$ well-defined aneurysm seen at the proximal A1 segment immediately after the ICA bifurcation that has ruptured leading to SAH and the consequent clinical presentation (Figure 1-B). To further evaluate the aneurysm and the full cerebrovascular tree, a digital subtraction angiography (DSA) cerebral angiogram was performed, again showing a 4 x 4 mm right $\mathrm{A} 1$ aneurysm and significant narrowing of the $\mathrm{M} 1$ segment of the right MCA, along with ipsilateral arterial-arterial collateral vessels, suggestive of a chronic, unilateral Moyamoya-like pathology (Figure 2). The dynamic pattern of filling and emptying with angiography was also very suggestive of a dissecting nature of that aneurysm. Calcium channel blockers were started, and the patient was placed under close supervision and blood pressure control monitoring in the intensive care unit (ICU); she was planned for an endovascular intervention on the next morning. On the same night, however, the patient experienced acute exacerbation of her headaches, and an urgent brain magnetic resonance imaging $(\mathrm{MRI})$ was done, revealing a right insular and caudate nucleus increased cortical thickening and signal abnormality on fluid-attenuated inversion recovery (FLAIR), suggesting hypoxic injury (Figure 3-A); also noted was the sign of chronic vaso-occlusive disease, the 'ivy sign' (Figure 3-B). In addition, MRI suggested an increase in the size of the aneurysm, explaining the exacerbation of headaches. During the endovascular procedure in the next morning, the pre-intervention angiographic images showed a significant 
increase in the size of the aneurysm, reaching $5.7 \times 4.5 \mathrm{~mm}$, intra-aneurysmal blood pooling, as well as severe stenosis of the ipsilateral M1 segment and stenosis of the ipsilateral supraclinoid ICA and A1 segment of the right ACA, suggestive of a vasospasm complicating the SAH and superimposed on the previously described MMD-related arterial stenosis (Figure 4). The dome and neck of the aneurysm, in addition to the right A1 segment, were occluded using 6 coils after confirming sufficient vascular supply of the ipsilateral anterior cerebral artery territory by the contralateral ACA. The post-operative neurological examination did not reveal any new deficit. The patient was monitored in the ICU for a total of 14 days and discharged home with an almost full neurologic recovery few days after the endovascular intervention. During her stay, transthoracic echocardiography was performed revealing a patent foramen ovale. The patient is planned for pial synangiosis in the near future to improve the compromised blood flow to the right MCA territory.

\section{Discussion}

The pathological architecture of the vascular tree and subsequent hemodynamic changes are important factors that contribute to the formation and determine the location of aneurysms in MMD patients. According to a recent study reporting aneurysms in children with MMD, aneurysms were almost equally distributed between the anterior (55.6\%) and posterior (44.4\%) circulations, the most common implicated vessels of which were the anterior choroidal artery and moyamoya vessels in the anterior circulation and posterior choroidal artery in the posterior circulation. ${ }^{6}$ In our patient, both the location and hemorrhagic type are peculiar. To our knowledge, none of the reported children with MMD had an ACA aneurysm, and only one patient presented with SAH from a posterior communicating artery. ${ }^{6}$ Our patient had a patent foramen ovale, which is consistent with reports of an association between congenital cardiac anomalies and MMD. ${ }^{2}$ 
Although not documented on histopathological examination, the aneurysm in our patient is most likely a dissecting aneurysm given the rapid increase in size, recurrent bleeding, and intraaneurysmal blood pooling noted on angiographic images; these characteristics were consistently reported in pseudoaneurysms of peripheral arteries of patients with MMD. ${ }^{7-10}$ Such aneurysms are more prone to rupture due to the persistent hemodynamic stress imposed by the vascular anomalies associated with MMD, ${ }^{11}$ and thus, prompt management is pivotal. In these patients, the management plan has two arms: acute, short-term stabilization and/or occlusion of the aneurysm and long-term, secondary prophylaxis against the development of new aneurysms and other MMD complications. The acute management plan may include surgical or endovascular interventions to exclude the ruptured aneurysm, ${ }^{6,12,13}$ whereas direct and indirect surgical bypass procedures such as superficial temporal artery-middle cerebral artery (STA-MCA) bypass, encephaloduroarteriosynangiosis (EDAS), and encephaloduroarteriomyosynangiosis (EDAMS $)^{14,}$ 15 are reserved for long-term management of MMD, especially in patients who had prior ischemic and hemorrhagic complications. We chose endovascular coiling rather than surgical clipping in this case because of the dissecting nature of the aneurysm, which necessitated parent artery along with pseudo-aneurysm occlusion. Conservative treatment was not an option due to the instability of the aneurysm, rapid increase in size, and recurrent bleeding. Pial synangiosis, a variation of EDAS, is planned for the long-term management of the condition. Indirect procedures may be easier to perform in children given the smaller diameter of the vessels and they provide similar long-term results in terms of revascularization; ${ }^{16}$ thus, higher rates of successful revascularization by indirect bypass are expected in pediatric MMD.

\section{Conclusions}


MMD-associated aneurysms are extremely rare in children, and hemorrhage may be the initial presentation of the disease. Prompt intervention (endovascular or surgical) is essential to exclude the ruptured aneurysm that is at risk of rebleeding due to the persistent hemodynamic stress. Long-term management with direct and/or indirect bypass should follow to decrease the risk of new flow-related aneurysm formation or development of other MMD-associated ischemic or hemorrhagic complications.

\section{Declaration of Interest: None}

\section{References}

1. Fukui M. Guidelines for the diagnosis and treatment of spontaneous occlusion of the circle of Willis ('moyamoya'disease). Research Committee on Spontaneous Occlusion of the Circle of Willis (Moyamoya Disease) of the Ministry of Health and Welfare, Japan. Clin Neurol Neurosurg. 1997;99: S238-240.

2. Scott R, Smith J, Robertson R, Madsen J, Soriano S, Rockoff M. Long-term outcome in children with moyamoya syndrome after cranial revascularization by pial synangiosis. $J$ Neurosurg. 2004;100(2 Suppl Pediatrics): 142-149.

3. Iwama T, Morimoto M, Hashimoto N, Goto Y, Todaka T, Sawada M. Mechanism of intracranial rebleeding in moyamoya disease. Clin Neurol Neurosurg. 1997;99: S187-190.

4. Kawaguchi S, Sakaki T, Morimoto T, Kakizaki T, Kamada K. Characteristics of intracranial aneurysms associated with moyamoya disease. A review of 111 cases. Acta Neurochir (Wien). 1996;138(11): 1287-1294. 
5. Takahashi J, Miyamoto S. Moyamoya disease: recent progress and outlook. Neurol Med Chir (Tokyo). 2010;50(9): 824-832.

6. Liu P, Lv X, Liu A, et al. Intracranial Aneurysms Associated with Moyamoya Disease in Children: Clinical Features and Long-Term Surgical Outcome. World Neurosurg. 2016;94: 513520.

7. Yuasa H, Tokito S, Izumi K, Hirabayashi K. Cerebrovascular moyamoya disease associated with an intracranial pseudoaneurysm. Case report. J Neurosurg. 1982;56(1): 131-134. 8. Miyake H, Ohta T, Kajimoto Y, Ogawa R, Deguchi J. Intraventricular aneurysms--three case reports. Neurol Med Chir (Tokyo). 2000;40(1): 55-60.

9. Park Y, Suk J, Kwon J. Repeated rupture of a middle meningeal artery aneurysm in moyamoya disease. Case report. J Neurosurg. 2010;113(4): 749-752.

10. Hamada J, Hashimoto N, Tsukahara T. Moyamoya disease with repeated intraventricular hemorrhage due to aneurysm rupture. Report of two cases. J Neurosurg. 1994;80(2): 328-331.

11. Burke G, Burke A, Sherma A, Hurley M, Batjer H, Bendok B. Moyamoya disease: a summary. Neurosurg Focus. 2009;26(4): E11-E11.

12. Narayan P, Workman M, Barrow D. Surgical treatment of a lenticulostriate artery aneurysm. Case report. J Neurosurg. 2004;100(2): 340-342.

13. Lévêque M, McLaughlin N, Laroche M, Bojanowski M. Endoscopic treatment of distal choroidal artery aneurysm. J Neurosurg. 2011;114(1): 116-119.

14. Baaj A, Agazzi S, Sayed Z, Toledo M, Spetzler R, van Loveren H. Surgical management of moyamoya disease: a review. Neurosurg Focus. 2009;26(4): E7-E7.

15. Pandey P, Steinberg G. Neurosurgical advances in the treatment of moyamoya disease. Stroke. 2011;42(11): 3304-3310. 
16. Fung L, Thompson D, Ganesan V. Revascularisation surgery for paediatric moyamoya: a review of the literature. Childs Nerv Syst. 2005;21(5): 358-364.

\section{Figure Legends}

Figure 1 - A. Grade II Subarachnoid hemorrhage localizing to the interhemispheric fissure, the right sylvian fissure and the right aspect of the suprasellar cistern B. CT angiography demonstrating a faint appearance of the supraclinoid segment of the right internal carotid artery and the M1 segment of the right middle cerebral artery, with a small aneurysm seen at the junction of the supraclinoid ICA and the right A1 segment (arrow).

Figure 2 - DSA diagnostic cerebral angiogram. Selective right internal carotid injection again demonstrating the right A1 aneurysm (arrows), with significant narrowing of the right M1 segment of the MCA and ipsilateral, arterial-arterial collateral vessels.

Figure 3 - A. MRI of the brain with diffusion weighted images. Restriction of diffusion indicating right insular and caudate nucleus ischemia (arrowheads). B. 'Ivy sign' (arrowheads) is seen on the FLAIR sequence of MRI.

Figure 4 - A. DSA pre-coiling cerebral angiogram showing an interval increase in the size of the right A1 aneurysm to reach 5.7 x $4.5 \mathrm{~mm}$ (arrow), intra-aneurysmal blood pooling (arrow), and cavernous transformation of the ipsilateral M1 segment. B \& C. 3D volume rendering images of the cerebral angiogram demonstrating the aneurysm (arrows).

Figure 5 - DSA cerebral angiogram: Post-endovascular cerebral coiling of the right A1 aneurysm using a total of 6 neurovascular coils (arrow) deployed to obliterate the dome and neck of the aneurysm as well as the parent A1 segment after confirming the presence of sufficient vascular supply of the ipsilateral anterior territory by the contralateral ACA. 